\title{
A Survey on Network Layer Multicast Routing Protocols for Mobile Ad Hoc Networks
}

\author{
Seetha. $\mathbf{R}^{1}$, Dr.R.Saravanan ${ }^{2}$ \\ School of Information Technology VIT University Vellore
}

\begin{abstract}
A Mobile Ad hoc Network (MANET) comprises set of mobile nodes which forms different networking infrastructure in ad hoc manner. Multicasting a group oriented communication approach plays a vital role in this mobile infrastructure less networks as it finds applications in major fields such as military warfare, emergency situations, Conventions etc. This paper surveys and discusses considerable number of multicast routing protocols of network layers as there exists several interesting tasks from formation of multicast session, cooperation of nodes to ending of multicast session, along with its advantages and disadvantages. It also provides various issues and challenges to be considered for designing such protocols for its use in MANET.
\end{abstract}

Keywords: Mobile Ad hoc Network (MANET), Multicast Routing Protocol, Tree based, Mesh based, Network layer, Source initiated, Receiver initiated.

\section{Introduction}

In computer networking, multicast is the delivery of a message or information from one host to many destination hosts. It involves transmission of data packets to a group of hosts identified by its destination address. Use of multicast in ad hoc networks improves its performance, efficiency, reduces cost of communication and is advantageous than multiple unicast because of scarce bandwidth and mobile nodes with limited energy resources. MANET, dynamic, autonomous, multi hop network have various outstanding features which includes rapid deployment, flexibility, mobility support, dynamic network topology. Thus multicast is important for MANET where more group communication occurs.

Wired network Internet protocol multicast routing protocol such as DVMRP, MOSPF, CBT, PIM do not perform well in ad hoc networks. These protocols establish a routing tree for a group of routing nodes for a multicast session. Once the tree is established, packet sent traverses each node and each link only once. Such a tree structure is not suitable for ad hoc networks where the structure will break when the nodes moves away and needs to be reconstructed continuously as connectivity changes. Maintaining a routing tree for multicasting packets, in the underlying topology changing frequently, can earn substantial control traffic. Therefore multicast protocols for static wired networks cannot be used for ad hoc wireless networks.

\section{Motivation}

The major aim of this survey paper is to provide the basic idea of multicasting and its protocols for network layers, its issues and challenges in MANET. The multicast routing protocols discussed are classified based on its structure used, operations, maintenance mechanism and functionalities. The paper is categorized as follows: section 3 discusses the multicast protocol architecture; section 4 discusses briefly classification and working mechanism of multicast routing protocols for network layers, section 5 gives summary and conclusion and finally in section 6 issues and challenges for designing multicast protocol is discussed as a part of future work.

\section{Architecture reference model for multicast routing protocol}

The multicasting in ad hoc networks has three layers in the network protocol stack:

- Medium Access Control (MAC) layer: It provides transmission and reception of packets to the above layers and also arbitrates access to the channel. MAC layer has three modules:

- Transmission module: includes arbitration module and schedules transmission.

- Receiver module: receives packets for upper layers.

- Neighbor list handler: maintains list of all neighbor nodes.

- Routing layer: This layer is responsible for forming and maintaining the unicast session/ multicast group. It uses the following modules:

- Unicast routing information handler

- Multicast information handler

- Forwarding module: Forwards packets either to the neighboring node or application layer based on the information provided by the multicast information handler. 
- Tree or Mesh construction module: This module constructs multicast topology.

- Session maintenance module: handles link breaks.

- $\quad$ Route cache maintenance module: updates and maintains information about the packets routed.

- Application layer: utilizes routing layer services to satisfy multicast application requirements. The modules used:

- Data packet transmit/receive controller.

- Multicast session initiator/terminator.

\section{Multicast Routing Protocols}

\subsection{Issues and challenges for Multicast protocol Design in Ad hoc Networks:}

Scarce bandwidth of ad hoc networks, rapidly moving nodes with limited resources (battery power, memory usage), hidden terminal problem and security concerns remains a great challenge for multicast protocols design. In addition to these challenges there are several issues to be considered but not limited to robustness (link stability), efficiency (ratio of total number of data packets received by receivers to the total number of packets (data and control) transmitted in the network, control overhead (control packets exchanged), Quality of service (throughput, delay, delay jitter, and reliability), dependency on unicast routing protocol, Resource management (battery power, memory).

\subsection{Classification of Multicast Routing protocol (MRP):}

- Based on its function: MRP is broadly classified into two categories as Application Specific and Application generic protocols.

- MRP on different layers of protocol stack: MAC layer provides reliable end - to -end communication. Network layer concentrates on routing and the Application layer provides easy deployment of protocol and creates a virtual topology which hides routing complications.

- Application generic protocols can be further classified on different dimensions:

- Based on structure constructed: Ad hoc multicast routing protocols uses two topology approaches which are classified as tree based and mesh based. Tree based multicast protocols are further divided into source tree based and shared tree based. In source tree based the source node forms the root of the tree whereas in the shard tree based multiple sources share a single tree with a core node at its root. The source tree based multicast protocols handles distribution of loads efficiently and the shared tree based multicast protocols offers scalability but the single point of failure affects the performance of multicast protocols due to is dependency on the core node. Mesh based multicast protocols are robust as there exists multiple paths between the source and destinations.

- Based on initialization of multicast session: The formation of multicast group can be initiated either by the source node or by the destination (receiver) node depending on which the multicast protocols can be called as source initiated multicast protocol or receiver initiated multicast protocol respectively.

- Based on maintenance mechanism: Multicast topology formed is maintained either by soft state approach (Proactive) or hard state approach (Reactive). In soft state approach the link between the nodes (source, receiver, and forwarding) is maintained by sending refreshing control packets periodically thus resulting in high packet delivery ratio with more control overheads. In hard state approach control packets are flooded only when link failure is detected thus minimizes the cost of overhead which leads to low packet delivery ratio.

\subsection{Tree based Multicast routing protocols:}

\subsubsection{Multicast Ad hoc On-Demand Distance Vector Routing Protocol (MAODV)}

Protocol Depiction: It is a shared tree based, receiver initiated multicast routing protocol. Uses hard state approach for maintaining the tree constructed. The MAODV protocol is an extension of AODV .It determines a multicast route on demand by using a broadcast route discovery mechanism. The first member of a multicast group becomes the leader of that group and is responsible for maintaining the multicast group sequence number and broadcasting this number to the multicast group. This is done through a group hellos (GRPHs) message. Node that wishes to join the group and having the address of group leader send a route request (RREQ) to the group leader otherwise broadcasts it. The member of the multicast group whose recorded sequence number greater than that in the RREQ replies with a route reply (RREP) which contains the current sequence number of multicast group distance of the replying node from the group leader. The receiver node joins the group by choosing a shortest path from all RREP packets received and on sending a multicast activation (MACT) message. The multicast tree is maintained using expanding ring search which uses RREQ, RREP, and MACT cycle.

Advantages: integrates unicasting and multicasting as a combined framework, loop free protocol.

Disadvantages: shared tree approach degrades the performance when single point of failure occurs. 


\subsubsection{Ad Hoc Multicast Routing Protocol utilizing Increasing ID-Numbers (AMRIS)}

Protocol Depiction: A shared tree based, source initiated, independent protocol and employs hard state approach for tree maintenance. AMRIS dynamically assigns every node of multicast tree an ID number known as msm-id. The node with the smallest msm-id, called the Sid forms the root of the multicast delivery tree root. For multiple senders, the sender with smallest msm-id becomes the Sid. Sid initiates the multicast session by broadcasting a NEW-SESSION message. The NEWSESSION message has the Sid's msm-id and the routing metrics. Neighbor nodes on receiving this message generate their own msm-id, which is larger than that specified in the NEW-SESSION message. The nodes then rebroadcast the NEW-SESSION message with their own msm-ids. A node wanting to join the multicast session sends a Join Request (JREQ) to the parent node with smallest msm-id. The parent, a member of desired multicast group, sends a Join Acknowledgment (JACK). Otherwise, the request is sent to the upstream node. When a link break occurs, the node with the larger msm-id is responsible for reconstruction. A node rejoins the tree by executing Branch Reconstruction (BR), which has two main subroutines, BR1 and BR2. BR1 is executed when the node has neighboring potential parent nodes which it attempts to join, and BR2 is executed when the node does not have any neighboring potential parent nodes where JREQ packet is flooded with some TTL value.

Advantages: simple, loop free, handles link breaks locally.

Disadvantages: use of beacon packets results in wastage of bandwidth, end-end delay in packet delivery and loss of packets.

\subsubsection{Bandwidth-Efficient Multicast Routing Protocol (BEMRP)}

Protocol Depiction: Source tree based, receiver initiated, autonomous multicast routing protocols repairs the link failure on demand (hard state). As the name indicates this protocol limits the use of bandwidth for delivering the packets by finding the next nearest forwarding node rather than flooding control packets to find the shortest path between source destination pair. The receiver node joins the group by flooding join control packet. The tree nodes receiving the join packets selects one packet with a smallest hop count and sends reply packet along the same path the selected join packet traversed. Link failures may be recovered by broadcastmulticast scheme (upstream links to its downstream along a new route) or local rejoin scheme (downstream node rejoins to upstream node).This protocol also determines optimized route by pruning unwanted forwarding nodes by sending a quit message.

Advantages: low bandwidth usage, easy to implement as it does not determine shortest paths.

Disadvantages: increase in distance between source and receiver increases probability of path breaks and hence results in low packet delivery ratio. Hard state approach delays packet delivery.

\subsubsection{Associatively- Based Ad hoc Multicast Routing (ABAM)}

Protocol Depiction: An on-demand source tree based, source initiated multicast routing protocol. The path of the tree from the source to the receiver is constructed based on stability and hence needs less reconfiguration. For tree construction the source floods multicast broadcast query packet (MBQ), the receiver replies with MBQreply packet along the stable path and the source sets up the tree by sending MC-setup packets to all the receivers who wish to join the multicast group. Intermediate nodes on receiving MBQ message appends their ID, associativity ticks (reflects the link stability by counting the beacon control packets received continuously from neighboring nodes) and other information (route relaying load, signal strength, power life) before rebroadcasting. Hard state approach is employed to maintain the multicast tree constructed and handles both leaf link break and branch link breaks effectively.

Advantages: achieves higher packet delivery ratio, low control overhead.

Disadvantages: increased hop distance between the source and the receiver makes the protocol less efficient and it is not scalable.

\subsubsection{Differential Destination Multicast Routing Protocol (DDM)}

Protocol Depiction: A stateless, source tree based, receiver initiated multicast routing protocol which depends on underlying unicast protocol and maintains the session using soft state approach. This protocol is more applicable for small group size. The source nodes inserts the destination address into the field, called the DDM block of the data packet, and unicasts it to the next node, using the underlying unicast routing protocol. A node receiving the DDM block data packet acquires the address of the next hop node and unicasts it. In this way, the data packets reach its destinations and thus the protocol avoids maintaining multicast states at the nodes. For maintaining the tree soft-state approach is employed where each node along the forwarding path remembers the destination address by storing it in the forwarding set. Thus caching this information, avoids listing all the destination addresses in every packet and hence the protocol is called the Differential Destination Multicast Routing protocol. 
Advantages: uses minimum memory resource as state information is not maintained, ensures security as the source node manages the group members.

Disadvantages: periodic control packet transmission results in significant consumption of bandwidth when there is an increase in no. of receivers, not scalable to large group size.

\subsubsection{Weight -Based Multicast Protocol (WBM)}

Protocol Depiction: Source tree based, receiver initiated, autonomous, multicast protocol which uses weight concept for a new multicast member to join the multicast tree. This weight concept allows the new multicast receiver to join the nearest node or the node nearest to the multicast source in the multicast tree. The receiver initiates the session by broadcasting JoinReq packet with time-to-live (TTL) entry. On receiving the request a tree node sends a Reply packet. The Reply packets arriving at a receiver node have the hop distance of receiver node from tree node that sent the Reply packet and hop distance of the tree node from source. Best pat is determined by means of parameter called joinWeight on considering the number of added forwarding nodes and also the hop distance between the source and destination. After receiving a number of Reply packets, the node maintains a best Reply, which is updated when new reply packets arrives. The best Reply minimizes the quantity, $Q=(1-$ joinWeight $) *($ hop distance of receiver from tree node -1$)+$ joinWeight $*$ (hop distance of receiver node from tree node + hop distance of tree node from Source node). A timer is set upon the receipt of first Reply packet. Once the timer expires, receiver node sends a JoinConf message along the path that the selected Reply has arrived. WBM uses localized prediction technique a soft state approach to maintain he multicast tree.

Advantages: Weight concept improves the efficiency of the protocol. Prediction based route mechanism prevents link breaks and increases packet delivery ratio.

Disadvantages: Prediction technique may not work well, in high fading environment. The joinWeight parameter depends on network load conditions and multicast group size.

\subsubsection{Multicast Routing Protocol Based on Zone Routing (MZRP)}

Protocol Depiction: Source tree based, Source initiated multicast protocol combines both proactive (Table driven approach, where each node maintains the topology of its zone) and on-demand approaches. Tree construction is initiated by the source node and each node is associated with a routing zone. The multicast tree nodes within the zone are maintained using proactive approach and tree construction is extended to other zones when necessary (on-demand). The source node refreshes the tree by sending Tree-refresh packets and any node not receiving it for some period of time is removed from the tree. When a link break is detected the isolated node within the zone joins the tree by unicasting Join packets to all nodes in the zone. If the node moves away from the zone joins the tree by sending JoinPropagate packet to the border nodes.

Advantages: Combines unicast (ZRP) and multicast routing protocol (MZRP), tunes through adapting zone sizes which are determined based on network load conditions.

Disadvantages: Considerable amount of time is taken by the receiver to join the session when the distance between the source and the receiver is large.

\subsubsection{Multicast Core-Extraction Distributed Ad Hoc Routing (MCEDAR)}

Protocol Depiction: MCEDAR a Source tree based multicast protocol combines the tree-based protocol and the Mesh-based protocol to provide efficiency and maintains the structure using hard state approach. It relies on CEDAR to construct the mesh called the mgraph as multicast routing infrastructure. CEDAR uses a core computation algorithm to create a minimum dominating set (MDS) of core nodes. Each core node announces its existence through a beacon control packet up to next three hops, and, thus each builds a virtual link identifying its near core nodes. Each non-core node located one hop away from at least one core node selects one of them as its dominator node. Either source or receiver can initiate the tree construction. When a non-core node wants a receiver to become a member of a multicast group, it requests its dominating core node, to perform the join operation. Core node broadcasts a JoinReq which contains address of the group (MA) the node wishes to join and the current joinID of the node corresponding to the multicast group. When a node that is not a member of multicast group (MA) receives the JoinReq, it forwards the message to its nearby core nodes. When an existing member of the multicast group receives the JoinReq, it sends a Join-Ack (MA, joinID of the replying node) only if its joinID is smaller than the joinID that arrives in the request. If its joinID is larger than the incoming one, it forwards the request just like a nonmember. An intermediate node receiving the Join-Ack message, decides whether to accept or reject it based on the robustness factor (R). Each mgraph has a parent set and a child set. Node accepting a Join-Ack, adds the upstream mgraph members to its parent set and the downstream members that are not already in its child set, forwards the Join-Ack to them adds them to its child set. When an intermediate node decides to reject a Join-Ack packet, it suppresses the Join-Ack packet and explicitly leaves from the upstream node so that its ID is removed from the upstream node's child set. The dominator accepts 
Join- Ack packets based the robustness factor $(\mathrm{R})$. If $\mathrm{R}=2$, only two Join-Ack packets are accepted by the dominator node rejecting other packets. The member on accepting a Join-Ack sets its joinID to the max (current joinID, arriving joinID) +1 . It then stamps the joinID of the Join-Ack with its new joinID.

Advantages: Robust and efficient, as a receiver node has multiple paths to a multicast tree.

Disadvantages: Due to bandwidth constraints, mobility of nodes and high channel access cost this protocol becomes less efficient and more expensive when used with small and sparsely distributed group environment. Increases control overhead, when nodes need to change their cores frequently in a high mobility environment. MCEDAR is also more complex (Tree-based and Mesh-based).

\subsubsection{Preferred Link-Based Multicast Protocol (PLBM)}

Protocol Depiction: PLBM a source tree based receiver initiated protocol employs hard state approach for tree maintenance. Here each node maintains two tables NNT (Neighbors Neighbors Table) maintains two-hop (on both streams) local network topology information and CT (Connect Table) for multicast tree information. Every node updates its NNT entry on receiving beacon packets that are transmitted periodically by every node. When a new member wants to join the group sends JoinConfirm message to the tree nodes in its NNT. Otherwise sends JoinQuery packet to one of the eligible nodes which are determined using Preferred Link-Based Algorithm (PLBA). Only the preferred nodes (first K eligible nodes in NNT) can process the received JoinQuery packet and forwards to eligible nodes starting the timer to receive JoinConfirm message from the receiver who wish to join the group. Each intermediate node updates its CT (path information both on upstream and downstream) on receiving JoinConfirm packet and marks itself as connected.

Advantages: The concept of the preferred link provides better adaptability and flexibility. Further, the use of 2hop local topology information provides efficient multicast routing.

The preferred list not only relies on neighboring nodes but also on other characteristics such as link delay, bandwidth, and link stability, network load.

Disadvantages: Transmission of beacon packets periodically considerably increases control overhead leading to wastage of bandwidth.

\subsubsection{Preferred Link-Based Unified Routing Protocol (PLBU)}

Protocol Depiction: An extension work of PLBM routes both unicast and multicast routing traffic simultaneously. PLBU has connect and reconfiguration phase. During connect phase the multicast tree is constructed and link breaks are reconfigured in reconfiguration phase. Source initiates connect phase for unicast routing whereas receiver initiates for multicast routing. It handles both unicast and multicast traffic in the same manner but for multicasting the multicast connectivity is confirmed to one of the tree nodes as there may be many RouteReply packets received from many tree nodes. PLBU makes use of route cache which reduces control overhead. The key information maintained by PLBU at the intermediate nodes is the two-hop information toward both streams which aids in quick reconfiguration of broken paths.

Discussion: Unifies unicast routing and multicast routing which reduces complexity and memory requirements in resource constrained ad hoc environment. Multicast session paths can be used for unicast session thus reducing control overhead in the network and hence PLBU is suitable for practical networks.

\subsubsection{Multicast for Ad Hoc Networks with Swarm Intelligence (MANSI)}

Protocol Depiction: MANSI utilizes swarm intelligence technique for multicast routing problem. Swarm intelligence deals with complex behaviors arising from very simple individual behaviors and interactions, often observed in nature, especially among social insects such as ants and honey bees. Each individual (for example an ant) has little intelligence and simply follows basic rules using local information obtained from their environment, global optimization objectives emerge when ants work collectively as a group. Similarly, MANSI utilizes small control packets that stores information at every visited nodes which is used later by other control packets. MANSI implements a shared tree-based approach for multicast connectivity among the members through a designated core node. Tree construction is initiated by the core node by announcing its being to others by flooding CoreAnnounce packet. Then the member nodes on receiving this announcement packet establish multicast tree by sending a Jreq back to the core via the reverse path. Intermediate nodes receiving a Jreq addressed to them become forwarding nodes of the multicast group accepts and rebroadcasts non-duplicated data packets. To maintain the links established and allow new members to join, the core floods CoreAnnounce packets periodically, as long as there are data packets to be sent. As an effect, these forwarding nodes form a mesh structure that connects the group members, while the core serves as a central point for tree creation and maintenance. MANSI tries to establish connectivity using minimum number of nodes and thus, nodes tend to choose paths that are partially shared by others to reduce the number of the forwarding nodes. Periodic exploration messages deployed by members to search for new forwarding nodes are replied by active 
forwarding members. If the cost of the new path is lower for the intermediate and requesting nodes, the requester switches to the new route leaving the old one.

Discussion: The concept of swarm intelligence reduces the number of nodes required to establish multicast routing tree. However, the path between the multicast member and forwarding set to the designated core need not be the shortest. MANSI employs a mesh-based approach to increase redundancy by allowing packets to be forwarded over more than one path, thereby increasing packet delivery ratio. In MANSI, group connectivity is made more efficient by allowing members to share common paths to the core with other members to reduce the total cost of forwarding data packets. Thus MANSI can be applied to many variations of multicast routing problems for ad hoc networks, such as load balancing, secure routing, and energy conservation.

\subsubsection{Adaptive Shared-Tree Multicast Routing Protocol (ASTM)}

Protocol Depiction: This protocol combines the features of source-tree-based and shared-tree-based. The shared-based tree rooted at Rendezvous Point (RP) is constructed initially by receivers by periodically sending JoinReq packets to the RP. The JoinReq includes forward list, which is initially set to include all senders. Sources send multicast data packets to the RP, and the RP forwards it to the receivers. The source sends the multicast data packet to RP which then forwards to desired receivers if the protocol is operating in the unicast shared mode. In multicast shared mode intermediate forwarding forwards the multicast data packets if it directly receives from the source nodes, if the packets are received from RP intermediate nodes discards it to avoid duplication of data packets. ASTM also allows sources to send multicast data packets directly to a receiver member without directing to RP when the distance (hop counts) between the source and receiver is small compared to the distance of RP and hence the name adaptive multicast (adaptive per source multicast routing). Thus ASTM supports switching between the shared tree and the per source tree depending on the distance between the source and the receiver.

Advantages: Adaptive nature of tree configuration makes it scalable (shared tree based) and increases packet delivery ratio (source tree based). Reduced control overhead (shared tree based)

And better throughput (shortest path between sender and receiver).

Disadvantages: Failure of RP affects multicast session resulting in packet losses.

\subsection{Mesh Based Multicast Routing Protocols:}

\subsubsection{On-Demand Multicast Routing Protocol (ODMRP)}

Protocol Depiction: A source-initiates the formation of mesh for multicast session. The mesh contains set of nodes called forwarding nodes to forward data packets. The source floods JoinReq packet periodically to form the mesh and the receivers joins the multicast session by sending JoinReply control packets via reverse shortest path. Multicast mesh is maintained by sending JoinReq control packets periodically. Nodes which want to leave the group just stops responding to JoinReq packet.

Advantages: Soft approach maintenance exhibits robustness.

Disadvantages: Increases control overhead, duplication of packets along multiple paths reduces efficiency and ODMRP suffers from scalability problem.

\subsubsection{Dynamic Core-Based Multicast Routing Protocol (DCMP)}

Protocol Depiction: Source initiated mesh based protocol and has three sources namely active source, passive source and core active sources. Each passive source has core active sources associated with it and acts as a proxy for the passive source by forwarding data for it over the mesh constructed by flooding JoinReq packets. Core active sources are the ones that floods JoinReq control packets on behalf of some passive sources. Active and core active sources initiates mesh creation and sends the data packets over the mesh whereas the passive nodes simply forwards it to its proxy nodes. Passive sources are determined by Maxhop distance (say 2) between them and core active nodes and the number of passive sources is limited by MaxPassSize to ensure robustness. Mesh maintenance is done by soft state approach.

Advantages: Scalable, high packet delivery ratio, decreased control overhead thus overcomes the limitations of ODMRP.

Disadvantages: The values associated to the parameters MaxHop and MaxPassSize depends on network traffic, group size and number of sources. Performance is degraded due to single point of failure.

\subsubsection{Forwarding Group Multicast Protocol (FGMP)}

Protocol Depiction: A receiver advertising mesh based multicast routing protocol employs forwarding group concept. It differs from ODMRP in mesh creation initiation. Receivers create the mesh by flooding JoinReq packets. On receiving these packets, each source updates it member table which contains IDs of all receivers and creates forwarding table. The forwarding table created is forwarded to the receivers. The path between source 
and receiver is established when the forwarding table reaches the receiver. FGMP applies soft state approach by flooding JoinReq packets on regular intervals to maintain the mesh.

Advantages: Robust and useful routing protocol when the numbers of senders are greater than the receivers.

Disadvantages: Increase in control overhead due to soft state approach.

\subsubsection{Neighbor Supporting Ad Hoc Multicast Routing Protocol (NSMP)}

Protocol Depiction: Mesh based source initiated multicast routing protocol employs selective and localized forwarding of packets. Source node creates the mesh by flooding Flood-Req control packets over the network. Receiver node receiving the control packets replies with Rep packet and establishes the route. Mesh is maintained by each source node which transmits Local-Req packets periodically that are forwarded by mesh nodes. The receiver nodes that are not within the range of multicast mesh may join the mesh by flooding MemReq (Member Request). Any multicast node may reply to the request with route discovery packet. When multiple route discovery packets arrives at receiver node, NSMP uses relative weight metric given as ((1$\alpha)^{*}$ Count of forwarding nodes from source to current node $+\alpha^{*}$ count of non-forwarding nodes from source to current node) to select a route for the new receiver node. The path with the lowest value is chosen for relative weight metric and $\alpha$ value depends on network load conditions. For mesh partitions group leaders among $\mathrm{t}$ sources is selected and is responsible for flooding Flood-Req for every Flood-Period interval.

Advantages: Reduced control overhead, high packet delivery ratio, use of relative weight metric for a node to join the multicast session improves efficiency of the protocol.

Disadvantages: Relative weight metric value changes as the load in the network varies and hence it must be made adaptive.

\subsubsection{Core-Assisted Mesh Protocol (CAMP)}

Protocol Depiction: Mesh based receiver initiated multicast routing protocol uses core node in the mesh for efficient bandwidth utilization. CAMP uses CAM (Core-to-group address) table which contains core ID. A receiver joins the shared mesh by getting core ID form CAM table and unicasts JoinReq packet to core node. The packets are forwarded using underlying unicast routing protocol. A mesh node sends back Ack packet to the receiver and hence it becomes the part of multicast group. CAMP establishes shortest paths by sending HeartBeat or PushJoin to the nodes. CAMP also supports sender only node to join simplex mode where data flows along only one direction. Mesh is maintained by employing hard state approach.

Advantages: Reduced control overhead exhibiting high packet delivery ratio.

Disadvantages: Failure of core nodes results in significant packet loss.

\subsubsection{Source Routing-Based Multicast Protocol (SRMP)}

Protocol Depiction: SRMP is an on-demand source initiated mesh based multicast routing protocol. SRMP uses Forwarding group (FG) concept to establish a mesh for each multicast session. SRMP employs source routing mechanism defined in the Dynamic Source Routing (DSR) protocol and avoids channel overhead and improves scalability. It deals with two important issues in solving the multicast routing: path availability concept and Long battery life path. When a source node wishes to join the group, broadcasts a Jreq packet to its neighbors, invokes a route discovery procedure for multicast group. The Jreq packet transmitted has the ID of source node, multicast group ID, and a Sequence number field which is set by the source node for each Jreq packet. Sequence number field is used to detect packet duplication. A first multicast receiver receiving Jreq packet, stores the multicast routing information, and checks its Neighbor Stability Table for stability information (association stability, link signal strength, and link availability) among its neighbors. Battery life is also verified taking into account the power required in transmitting to each neighbor. A neighbor is selected as an FG node if the four selection metrics satisfy their predefined thresholds. The receiver then sends a Jrep packet to each FG node, setting its type as "member node" in the Neighbor Stability Table. If no neighbor nodes satisfying the predefined thresholds are available, the node with the best metrics among all the neighbor nodes will be selected as an FG node. Thus the route is constructed and a multicast source node sends multicast data toward the multicast group nodes. Any node wishing to leave the multicast session just sends Leave-Group message to its neighbor nodes.

Advantages: SRMP establishes stable multicast path links maximizing the lifetime of multicast session routes, ensures reliability and robustness, and results in less power consumption. On-demand route discovery mechanism and link break detection minimizes control overhead saving bandwidth and network resources.

Disadvantages: The value of the four metrics used in selecting the paths are not globally constant and varies with different network load conditions. Hence the four metrics must be made adaptive to dynamic network traffic. 


\section{Summary and Conclusion}

Table-1 summarizes the characteristics of multicast routing protocols discussed for quick reference. Thus this paper presents a comprehensive survey of considerable number of network layer multicast routing protocols and provides a broader understanding. It also provides information about Multicast Architecture Reference model. Various issues and challenges that are to be considered in designing multicast routing protocols for Ad Hoc networks aids in research direction.

\section{Future Work}

The challenges faced by multicast routing protocols for ad hoc wireless networks are more complex than faced for wired networks. Some of the parameters that affect multicast routing in ad hoc wireless networks include Packet delivery ratio, total overhead, efficiency, average end-to-end latency, average throughput. Reliable multicasting with scarce bandwidth and limited battery power resources still remains a greater challenge for multicast routing protocols in high mobile ad hoc networks. Another greater concern for multicast routing protocol in ad hoc wireless is security. Other issues include load balancing, resource management. The research topics can b extended to but not limited to interoperability of protocols with other networks, interaction among unicast, multicast and broadcast applications, Integration of quality of service, network coding for better resource utilization and channel capacity. Designing a multicast routing protocol that takes all these issues into consideration is highly complex. One better possible solution would be an adaptive approach to multicast routing protocol.

\begin{tabular}{|c|c|c|c|c|c|c|c|c|}
\hline MRP & $\begin{array}{l}\text { Multicast } \\
\text { Topology }\end{array}$ & Initialization & $\begin{array}{l}\text { Autonomous } \\
\text { or } \\
\text { Dependent }\end{array}$ & $\begin{array}{l}\text { Maintenance } \\
\text { Approach }\end{array}$ & $\begin{array}{l}\text { Loop } \\
\text { Free }\end{array}$ & $\begin{array}{l}\text { Flooding } \\
\text { of } \\
\text { control } \\
\text { packets }\end{array}$ & $\begin{array}{l}\text { Periodic } \\
\text { Control } \\
\text { Messagin } \\
\mathrm{g}\end{array}$ & $\begin{array}{l}\text { Control } \\
\text { Overhea } \\
\text { d }\end{array}$ \\
\hline $\begin{array}{l}\text { MAOD } \\
\text { V }\end{array}$ & Shared-Tree & Receiver & $\begin{array}{l}\text { Dependent on } \\
\text { unicast based } \\
\text { AODV }\end{array}$ & Hard state & Yes & Yes & Yes & Low \\
\hline AMRIS & Shared-Tree & Source & Autonomous & Hard state & Yes & Yes & Yes & Low \\
\hline $\begin{array}{l}\text { BEMR } \\
\text { P }\end{array}$ & Source-Tree & Source & Autonomous & Hard state & Yes & Yes & No & Low \\
\hline ABAM & Source-Tree & Source & Autonomous & Hard state & Yes & Yes & No & Low \\
\hline DDM & Source-Tree & Receiver & $\begin{array}{ll}\text { Depends on } \\
\text { unicast } \\
\text { routing } \\
\text { protocol }\end{array}$ & Soft state & Yes & Yes & Yes & Low \\
\hline WBM & Source-Tree & Receiver & Autonomous & Hard state & Yes & Yes & No & Low \\
\hline MZRP & Source-Tree & Source & $\begin{array}{l}\text { Unicast based } \\
\text { ZRP }\end{array}$ & Hard state & Yes & Yes & Yes & Low \\
\hline $\begin{array}{l}\text { MCED } \\
\text { AR }\end{array}$ & $\begin{array}{l}\text { Source-Tree } \\
\text { over mesh }\end{array}$ & $\begin{array}{ll}\text { Source } & \text { or } \\
\text { Receiver } & \end{array}$ & $\begin{array}{l}\text { Unicast based } \\
\text { CEDAR }\end{array}$ & $\begin{array}{l}\text { Hard state } \\
\text {. }\end{array}$ & Yes & Yes & No & High \\
\hline PLBM & Source-Tree & Receiver & $\begin{array}{l}\text { Unicast based } \\
\text { PLBR }\end{array}$ & Hard state & Yes & No & Yes & High \\
\hline PLBU & Source-Tree & Receiver & $\begin{array}{l}\text { Unicast based } \\
\text { PLBR }\end{array}$ & Hard state & Yes & No & Yes & Low \\
\hline MANSI & $\begin{array}{l}\text { Shared-Tree } \\
\text { over mesh }\end{array}$ & Core initiated & Autonomous & Hard state & Yes & Yes & Yes & Low \\
\hline
\end{tabular}


A survey on Network layer Multicast Routing Protocols for Mobile Ad hoc networks

\begin{tabular}{|l|l|l|l|l|l|l|l|l|}
\hline ASTM & $\begin{array}{l}\text { Combination } \\
\text { of shared \& } \\
\text { source tree }\end{array}$ & receiver & $\begin{array}{l}\text { Depends on } \\
\text { unicast } \\
\text { routing } \\
\text { protocol }\end{array}$ & Soft state & Yes & Yes & Yes & High \\
\hline ODMRP & Mesh & Source & Autonomous & Soft State & Yes & Yes & Yes & High \\
\hline DCMP & Mesh & Source & Autonomous & Soft State & Yes & Yes & Yes & Low \\
\hline FGMP & Mesh & Receiver & Autonomous & Soft State & Yes & Yes & Yes & Low \\
\hline NSMP & Mesh & Source & Autonomous & Soft State & Yes & Yes & Yes & Low \\
\hline CAMP & Mesh & $\begin{array}{l}\text { Source or } \\
\text { Receiver }\end{array}$ & $\begin{array}{l}\text { Dependent } \\
\text { on any } \\
\text { proactive } \\
\text { protocol }\end{array}$ & Hard State & Yes & No & No & Low \\
\hline SRMP & Mesh & Source & $\begin{array}{l}\text { Depends on } \\
\text { unicast based } \\
\text { DSR Hard State }\end{array}$ & Yes & No & No & Low \\
\hline
\end{tabular}

Table 1 - Comparison and Summary table for Multicast Routing Protocol

\section{References}

[1] IETF MANETWorking Group, http://www.ietf.org.

[2] C.-K. Toh, Ad Hoc Mobile Wireless Networks: Protocols and Systems, Prentice-Hall, Englewood Cliffs, NJ, USA, 2002.

[3] C. Perkins, Ad-Hoc Networking, Addison-Wesley, Reading, Mass, USA, 2000.

[4] C.-C. Chiang, M. Gerla, and L. Zhang, "Adaptive shared tree multicast in mobile wireless networks," in Proceedings of the IEEE Global Telecommunications Conference (GLOBECOM '98), vol. 3, pp. 1817-1822, 1998.

[5] C.-C. Shen and C. Jaikaeo, "Ad hoc multicast routing algorithm with swarm intelligence," Mobile Networks and Applications, vol. 10 , no. 1 , pp. $47-59,2005$

[6] C.W.Wu, Y. C. Tay, and C.-K. Toh, "Ad hocMulticast Routing protocol utilizing Increasing id-numberS (AMRIS)," draftietfmanet-amris-spec-00.txt, 2000.

[7] C.-K. Toh, G. Guichal, and S. Bunchua, "ABAM: on-demand associativity-based multicast routing for ad hoc mobile networks," in Proceedings of the IEEE Vehicular Technology Conference (VTC'00), vol. 3, pp. 987-993, 2000. [8] T. Ozaki, J. B. Kim, and T. Suda, "Bandwidth-efficient multicast routing for multihop, ad-hoc wireless networks," in Proceedings of the 20th Annual Joint Conference of the IEEE Computer and Communications Societies (INFOCOM'01), vol.2, pp. 1182-1191, 2001.

[9] J. J. Garcia-Luna-Aceves and E. L. Madruga, "The coreassisted mesh protocol," IEEE Journal on Selected Areas in Communications, vol. 17, no. 8, pp. 1380-1394, 1999.

[10] L. Ji and M. S. Corson, "Differential destination multicast- a MANET multicast routing protocol for small groups," in Proceedings of the 20th Annual Joint Conference of the IEEE Computer and Communications Societies (INFOCOM'01), vol. 2, pp. 1192-1201, 2001 .

[11] S. K. Das, B. S. Manoj, and C. S. R. Murthy, "A dynamic core based multicast routing protocol for ad hoc wireless networks," in Proceedings of the International Symposium on Mobile Ad Hoc Networking and Computing (MobiHoc '02), pp. 24-35, 2002.

[12] C.-C. Chiang, M. Gerla, and L. Zhang, "Forwarding Group Multicast Protocol (FGMP) for multihop, mobile wireless networks," ACM-Baltzer Journal of Cluster Computing, vol. 1, no. 2, pp. 187-196, 1998

[13] R. S. Prasun Sinha and V. Bharghavan, "MCEDAR: multicast core-extraction distributed ad hoc routing," in Proceedings of the Wireless Communications and Networking Conference, vol. 3, pp. 1313-1317, 1999

[14] E. M. Royer and C. E. Perkins, "Multicast ad hoc on demand distance vector (MAODV) routing," Internet-Draft, draft-ietfdraftmaodv-00.txt, 2000

[15] X. Zhang and L. Jacob, "MZRP: an extension of the zone routing protocol for multicasting in MANETs," Journal of Information Science and Engineering, vol. 20, no. 3, pp. 535- 551, 2004.

[16] S. Lee and C. Kim, "Neighbor supporting ad hoc multicast routing protocol," in Proceedings of the ACM International Symposium on Mobile Ad Hoc Networking and Computing (MobiHoc '00), pp. 37-44, 2000.

[17] S.-J. Lee,W. Su, andM. Gerla, "On-demand multicast routing protocol in multihop wireless mobile networks," Mobile Networks and Applications, vol. 7, no. 6, pp. 441-453, 2002.

[18] R. S. Sisodia, I. Karthigeyan, B. S. Manoj, and C. S. R. Murthy, "A preferred link based multicast protocol for wireless mobile ad hoc networks," in Proceedings of the IEEE International Conference on Communications (ICC '03), vol. 3, pp. 2213- 2217, 2003.

[19] H. Moustafa and H. Labiod, "SRMP: a mesh-based protocol for multicast communication in ad hoc networks," in Proceedings of the International Conference on Third Generation Wireless and Beyond 3Gwireless, pp. 43-48, May 2002.

[20] S. K. Das, B. S. Manoj, and C. S. R. Murthy, "Weight based multicast routing protocol for ad hoc wireless networks," in Proceedings of the IEEE Global Telecommunications Conference (GLOBECOM '02), vol. 1, pp. 117-121, 2002.

[21] C. S. R. Murthy and B. S. Manoj, Ad Hoc Wireless Networks: Architectures and Protocols, Prentice-Hall, Upper Saddle River, NJ, USA, 2004.

[22] L. Junhai, X. Liu, and Y. Danxia, "Research on multicast routing protocols for mobile ad-hoc networks," Computer Networks, vol. 52, no. 5, pp. 988-997, 2008.

[23] R. S. Sisodia, B. S. Manoj, and C. S. R. Murthy, "A preferred link based routing protocol for wireless ad hoc networks,"Journal of Communications and Networks, vol. 4, no. 1, pp. 14-21, 2002.

[24] T. Ballardie, P. Francis, and J. Crowcroft, "Core based trees (CBT)," ACM SIGCOMM Computer Communication Review, vol. 23, pp. 85-95, 1993.

[25] M. S. Corson and J. Macker, "Mobile Ad hoc Networking (MANET): Routing Protocol Performance Issues and Evaluation Considerations," Internet Engineering Task Force, 1999.

[26] Osamah S. Badarneh andMichel Kadoch, "Multicast Routing Protocols in Mobile Ad Hoc Networks", EURASIP Journal on Wireless Communications and Networking Volume 2009. 